\title{
Electron Compton scattering on solids- a feasibility experiment on a PEELS system
}

\author{
Peter Schattschneider $\left({ }^{1}\right)$, Peter $\operatorname{Jonas}\left({ }^{1}\right)$ and Mathias $\operatorname{Mändl}\left({ }^{2}\right)$ \\ ( $\left.{ }^{1}\right)$ Inst. f. Angewandte u. Techn. Physik, TU Wien, A-1040 Vienna, Austria \\ $\left({ }^{2}\right)$ Inst. f. Angewandte Physik, Univ. Regensburg, D-8400 Regensburg, Germany \\ (Received November 09, 1990; accepted January 28, 1991)
}

\begin{abstract}
ECOSS (Electron Compton Scattering on Solids) is a powerful method in order to obtain the momentum density distribution of conduction electrons. Apart from the Bragg-Compton channel coupling which produces a strong and often irregular background, the main problem in ECOSS has been the low count rate at scattering angles where the impulse approximation is valid. Simulations showed that the Gatan 666 PEELS should be capable of producing spectra of sufficient accuracy within a dwell time of some $100 \mathrm{~s}$, when dark current and flat field corrections are applied. Preliminary experiments with $\mathrm{Al}$ prove that Compton spectra measured on a Gatan 666 attached to a Philips CM30 compare well with predicted ones. Extraction of the momentum anisotropy on an unprecedented momentum scale of 0.04 a.u. (i.e. $\sim 0.07 \AA^{-1}$ ) appears feasible.
\end{abstract}

\section{ECOSS.}

Electron-Compton scattering on solids (ECOSS) is inelastic scattering of fast electrons under a large scattering angle. As is the case in photon Compton experiments, the scattered particle loses energy (Compton shift), the energy loss for fixed angle being dependent on the initial momentum of the scatterer which is a core- or valence electron of the solid. Hence, the Compton profile in the loss spectrum is a measure of the momentum distribution of the scatterer in its ground state $[1,2]$.

ECOSS offers a number of advantages as compared to photon Compton experiments:

a) at typical angles the scattering cross section for electrons is by five orders of magnitude larger than for photons;

b) the brightness of modern electron sources surpasses that of photon sources by two orders of magnitude;

c) post specimen electron optical devices allow a much better momentum resolution than photon Compton scattering devices;

d) focussing of electrons onto the specimen allows spatial resolutions of some $\mathrm{nm}$, in principle, compared to $\mathrm{mm}$ in photon work.

From a), b) it is evident that the sensitivity of ECOSS enormously exceeds that of photon Compton scattering; the duration of an experiment can be reduced from days for photon scattering to 
fractions of hours.

The major drawback in ECOSS relates to a) above. The large cross section for scattering of electrons in matter brings about a huge background which easily overrides the Compton signal. Figure 1 is a typical electron Compton spectrum with a background-to-signal ratio $k=0.8$ which is a rather small values. Unfortunately, the background does not follow a simple law, in general, and background handling is therefore a delicate task. This problem has been discussed elsewhere [3-5]. Here we are concerned with the fact that even with the much higher scattering cross section for electrons, the count rate of Compton electrons at the detector is notoriously low. This is the reason why ECOSS experiments have been limited to a few trials [5-8].

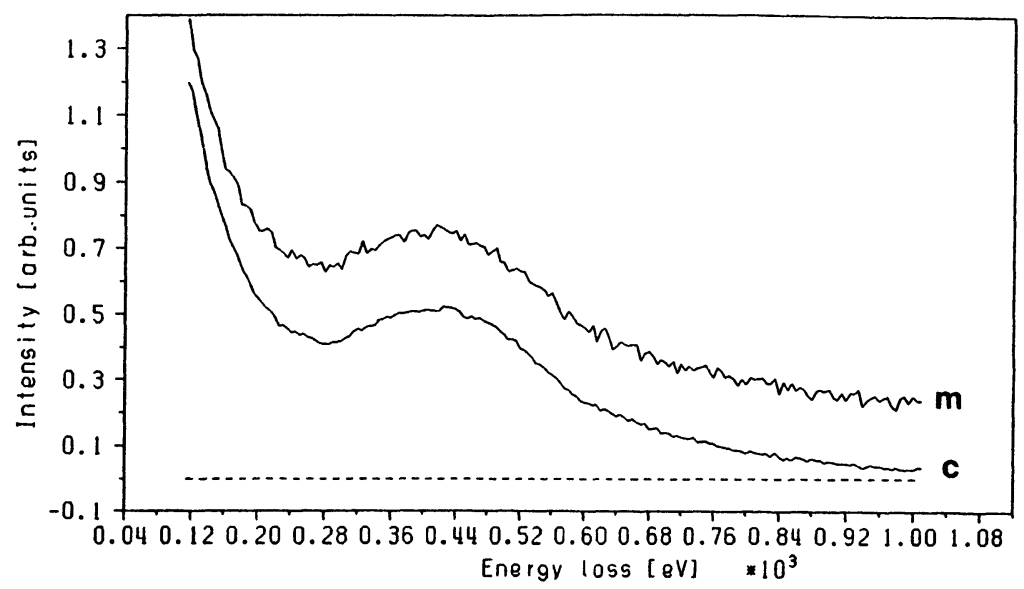

Fig. 1. - Compton spectrum of polycrystalline aluminium. (m): as measured on a GATAN 666 PEELS with $200 \mathrm{~s}$ dwell time; (c): after linear correction (Eq. (8)).

In the past decade the increasing availability of PEELS systems [9] has offered new possibilities for previously unfeasible energy loss experiments such as ECOSS.

As yet, the indirect detection of electrons via a YAG scintillator seems to be the method of choice for PEELS $[10,11]$. As a detector, linear PDAs $[10,11,15]$ or two-dimensional CCDs [12, $13,16]$ are used.

At count rates of some ten electrons per second, as expected in ECOSS (see Sect. 2), dark current may mask the useful Compton signal, and it is well known that PDAs produce a much higher dark current than CCDs. Therefore, a YAG-CCD combination should perform better for ECOSS. On the other hand, among the few commercially available EELS machines, the GATAN 666 which has a PDA seems more attractive for a number of reasons. So it is important to know whether or not the Gatan 666 is capable of producing ECOSS spectra of sufficient accuracy.

\section{What is typical for ECOSS.}

In the following, we give an estimate of parameters for ECOSS experiments, after which we calculate the necessary dwell times on a Gatan 666 for a given signal-to-noise ratio (SNR).

ECOSS needs peak energy losses of at least $4 \times E_{\mathrm{i}}$ where $E_{\mathrm{i}}$ is the ionisation energy of the electrons which are to be probed [2]. In $\mathrm{Al}$, we aim at the conduction and L-shell electrons. 
$E_{\mathrm{i}}=73 \mathrm{eV}$, and the Compton peak should then be at $E \geq 290 \mathrm{eV}$. From the free electron parabola

$$
E=\frac{\hbar^{2} q^{2}}{2 m}
$$

this corresponds to a wave number transfer of $q \approx 9 \AA^{-1}$. This transfer defines a scattering angle of $36 \mathrm{mrad}$ and $28 \mathrm{mrad}$ from the incident direction at $200 \mathrm{kV}$ and at $300 \mathrm{kV}$, respectively.

Assuming an energy resolution of $5 \mathrm{eV}$ we have

$$
\delta q=\frac{\mathrm{d} q}{\mathrm{~d} E} \delta E=\frac{q}{2 E} \delta E=0.08 \AA^{-1}
$$

much better than typically $0.4 \AA^{-1}$ for photon-Compton experiments. At $200 \mathrm{kV}$ this is $0.31 \mathrm{mrad}$, corresponding to a disk of $\sim 1 \mathrm{~mm}$ diameter in the diffraction plane, when the camera length is set to $3.5 \mathrm{~m}$. Hence, with a spot of that diameter which can be easily obtained with moderate condenser overfocus a fairly good match of the momentum resolution to the smallest spectrometer aperture of the Gatan spectrometer $(1 \mathrm{~mm})$ is achieved $\left({ }^{1}\right)$.

The expected count rate at the spectrometer entrance aperture is

$$
I=I_{0} \cdot \Delta \sigma \cdot N
$$

where $I_{0}$ is the incident rate, $\Delta \sigma$ is the partial differential cross section of $\mathrm{Al}$ for the energy and momentum windows set, and $N$ is the number of irradiated atoms in the specimen.

In selected area diffraction (SAD), working with a small selector aperture and a moderate condenser defocus, we measured a primary current density of $I_{0}=7 \mathrm{kA} / \mathrm{m}^{2}$ on the specimen, and the irradiated volume was a cylinder of $1 \mu$ diameter and $50 \mathrm{~nm}$ height. This makes $N=2.3 \times$ $10^{9} \mathrm{Al}$ atoms.

For the partial cross section, we start from

$$
\frac{\partial^{2} \sigma}{\partial E \partial \Omega}=\left(\frac{2 m e^{2}}{\hbar^{2} q^{2}}\right)^{2} \cdot \frac{\hbar^{2} q^{2}}{2 m E} f
$$

where $f=f_{\mathrm{c}}+f_{\mathrm{v}}$ is the generalized oscillator strength for core and valence electrons [19]. $f$ can be calculated e.g. by a hydrogenic model [14]. Eventually, for the resolution given above, $\left(\Delta E=5 \mathrm{eV}, \Delta \Omega=9 \times 10^{-8} \mathrm{sr}\right)\left({ }^{2}\right)$ and a scattering angle of $34 \mathrm{mrad}$ at $300 \mathrm{kV}$, we calculate a partial cross section in the Compton maximum:

$$
\Delta \sigma=\int_{\Delta E} \int_{\Delta \Omega} \frac{\partial^{2} \sigma}{\partial E \delta \Omega} \mathrm{d} E \mathrm{~d} \Omega=3 \times 10^{-31} \mathrm{~m}^{2} / \text { atom }
$$

The expected count rate is therefore

$$
I=I_{0} \cdot \Delta \sigma \cdot N \approx 30 \text { electrons } / \mathrm{s} .
$$

(1) The overall momentum resolution $\Delta q$ in an experiment is governed not only by the energy resolution given in equation (2), but also by the convergence angle of the illuminating system and by the spectrometer aperture. Very approximately, we have $\Delta q=\sqrt{3} \cdot \delta q$ for an optimum match between the three resolutionlimiting factors.

$\left({ }^{2}\right)$ Although the measurements described in section 4 were done at a dispersion of $2 \mathrm{eV} /$ channel, the resolution was not better than given because of the instabilities in the zero loss during a dwell time of $200 \mathrm{~s}$. 
For an SNR of $30(\approx 3 \%$ counting statistics) at peak value, assuming an ideal detector system, this would imply $30 \mathrm{~s}$ dwell time. Previous experiments [4] showed the background to be between 1 and 5 times the peak signal which would increase the necessary dwell time by a factor of 2 to 6 , still achievable in a single readout on the GATAN 666, collecting from 3600 to 32000 electrons in the intensity maximum.

The technical data sheet states DQE $>0.4$ at $10^{4}$ electrons/channel. Taking the lower bound and a large background, a dwell time of $\sim 450 \mathrm{~s}$ would be necessary. By carefully optimizing parameters such as scattering angle, background and incident current, a reduction by a factor of 5 appears feasible, allowing a Compton spectrum to be collected within some $100 \mathrm{~s}$ compared to some days in photon-Compton work.

It should be noticed that we used rather unfavourable parameters in the above estimate. On the other hand, the GATAN 666 data sheet does not explicitely state a dark current, and it might well be that with the unusually small count rates of ECOSS the given DQE is largely reduced due to the otherwise negligible dark current.

Therefore, we discuss the SNR and DQE in more detail, relying partly on the technical specification, partly on a paper giving some more information on the YAG-PDA detector [15] and partly on own measurements.

\section{Detective quantum efficiency and SNR.}

The SNR is given by

$$
\mathrm{SNR}=\frac{s t}{\sqrt{\sum \sigma_{i}^{2}}}
$$

where $s$ is the rate of the signal in question, given in primary incident electrons/time, $t$ is the dwell time, and the denominator contains all sources contributing to the noise. There are mainly four sources of noise in the present type of measurement:

- $\sigma_{\mathrm{c}}^{2}=s^{\prime} t$ is the variance due to counting statistics, $s^{\prime}=s(1+k)$ is the combined signal plus background rate, and $k$ is the background to signal ratio.

- $\sigma_{\mathrm{g}}=s^{\prime} t \cdot \delta G / G$ is the rms noise value due to the residual channel-to-channel gain variation $\delta G / G$ after software flatfield correction.

- $\sigma_{\mathrm{d}}^{2}=d t$ is the variance of the dark charge accumulated in the PDA but referred back to primary electrons. $d$ is an equivalent dark current expressed in primary electrons/s which would yield the given variance.

- $\sigma_{\mathrm{r}}$ is the combined reset, amplifier and A-D conversion noise of the system, again expressed in primary electrons.

Table I. - Parameters for DQE of the GATAN 666 and a YAG-CCD system.

\begin{tabular}{|l|c|c|c|}
\hline \multicolumn{1}{|c|}{ source } & $\delta G / G$ & $d$ [prim. el/s] & $\sigma_{\mathrm{r}}[\mathrm{prim} . \mathrm{el} / \mathrm{s}]$ \\
\hline GATAN 666 data sheet & $<0.01$ & not given & $<90$ \\
Krivanek et al. $[15]$ & 0.003 & 2.4 & 37 \\
measured & - & $<19$ & - \\
& & & \\
CCD & $0.004[9]$ & $0.45-5[17,18]$ & $3.3-10[16,18]$ \\
\hline
\end{tabular}


Factoring out terms in equation (6),

$$
\mathrm{SNR}=\sqrt{s^{\prime} t} \frac{1}{1+k} \sqrt{\frac{1}{1+s^{\prime} t(\delta G / G)^{2}+d / s^{\prime}+\sigma_{\mathrm{r}}^{2} / s^{\prime} t}} .
$$

The last term in the square-root is the DQE. It is worth noting that it not only depends on the number of accumulated electrons but also on the rate $s^{\prime}$.

In order to calculate the DQE of a detector, three parameters are needed: $\delta G / G, d, \sigma_{\mathrm{r}}$. These parameters are somewhat uncertain as can be seen from the table.

The dark current of the GATAN 666 was determined by measurement of a dark spectrum with a dwell time of 200 seconds and subsequent linear correction

$$
N_{\mathrm{c}}=\frac{N-D}{U-D}
$$

where $N, N_{\mathrm{c}}$ are the measured and corrected values, $D$ is a separately obtained dark spectrum, actually averaged over 4 spectra of $200 \mathrm{~s}$ dwell time each, and $U$ is a spectrum obtained by uniformly illuminating the PDA. Figure 2a shows the statistics in the dark spectrum, before and after correction. Figure $2 b$ is the distribution function of deviations from mean value for 100 channels. The statistical analysis yields a standard deviation of \pm 2.4 counts. For a conversion efficiency of 30 primary electrons/count this amounts to a variance of $\sigma_{\text {total }}^{2}=\sigma_{\mathrm{d}}^{2}+\sigma_{\mathrm{r}}^{2}+\sigma_{\mathrm{g}}^{2}=5200 e^{-}$. Taking [15] $\sigma_{\mathrm{r}}^{2}=37^{2}$ and $\delta G / G>0$, the dark current is $d<19$ electrons/s.

Results for the DQE of the GATAN 666 are shown in figures 3,4 . Note that at usual rates of some $10^{3}$ counts/s, dark current is not a problem et all. and DQE(N) looks very similar to that given in [15]. At lower rates, DQE becomes dark current dominated and drops significantly at a critical rate of less than 100 counts/s as can be seen from figure 4. ECOSS lies "on the edge" of the DQE breakdown.

Figure 5 is the calculated SNR as a function of the count rate with time as a parameter. The background to signal ratio was assumed to be 2 . The experimental ECOSS range is marked. At some $100 \mathrm{~s}$ dwell time, SNRs of 30 and more can be obtained.

\section{Experimental.}

In order to test predictions, we measured the Compton spectrum of a $50 \mathrm{~nm}$ thin polycrystalline Al-film with a Philips CM30 at $300 \mathrm{kV}$. The dwell time was $200 \mathrm{~s}$, and the scattering angle and accuracy was set according to the estimate given in section 2 . Results are shown in figure 1 . Note the strong background and the significant improvement of the noise after linear correction.

After background subtraction, the theoretical profile is shown superimposed in figure 6 . The discrepancies on the low energy side are caused by the strong influence of the L-edge. The overall fit is quite good, and it is to be expected that by choosing optimum parameters accurate information on the momentum distribution in the solid, with a resolution of better than $0.07 \AA^{-1}$, corresponding to an energy resolution of $2.5 \mathrm{eV}$ and an optimum match of convergence angle to the spectrometer entrance aperture can be obtained by ECOSS.

The experimentally determined SNR in the Compton profile figure 6 is 64 . This is better than the value of 45 predicted by equation (7) for the present experimental conditions ( $t=200 \mathrm{~s}, k=$ $0.8, s^{\prime}=49$ ). One reason for this discrepancy is certainly the fact that we used an upper bound for the dark current. Lowering that value would increase the estimated SNR. Future experiments should deal with the dark current problem in more detail. 


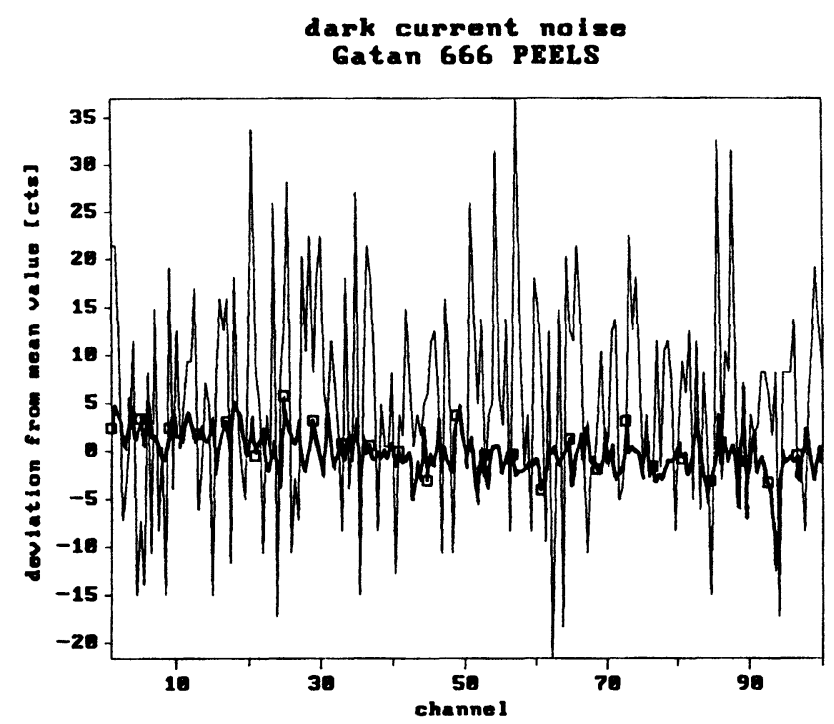

a)

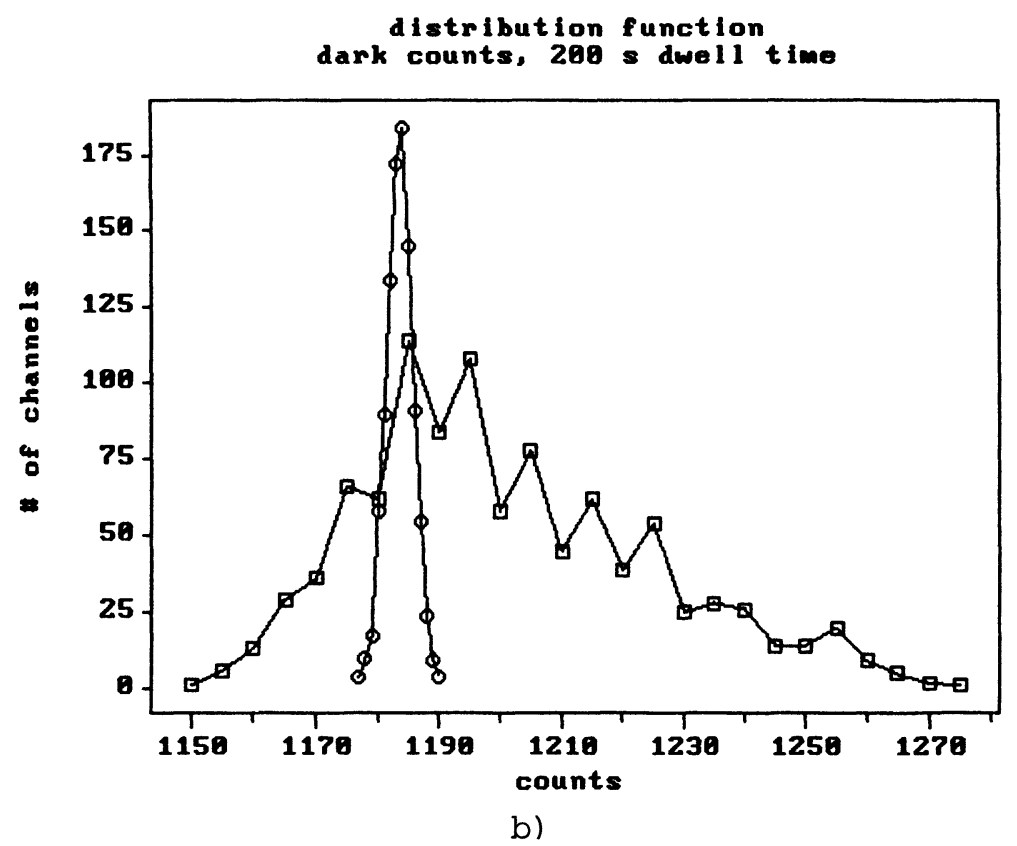

Fig. 2. - (a) Noise in 100 channels dark spectrum, before (thin line) and after ( $\square$ ) linear correction by equation (8). (b) Distribution function of the noise in figure $2 a(\square)$ as measured; (o) after linear correction. 


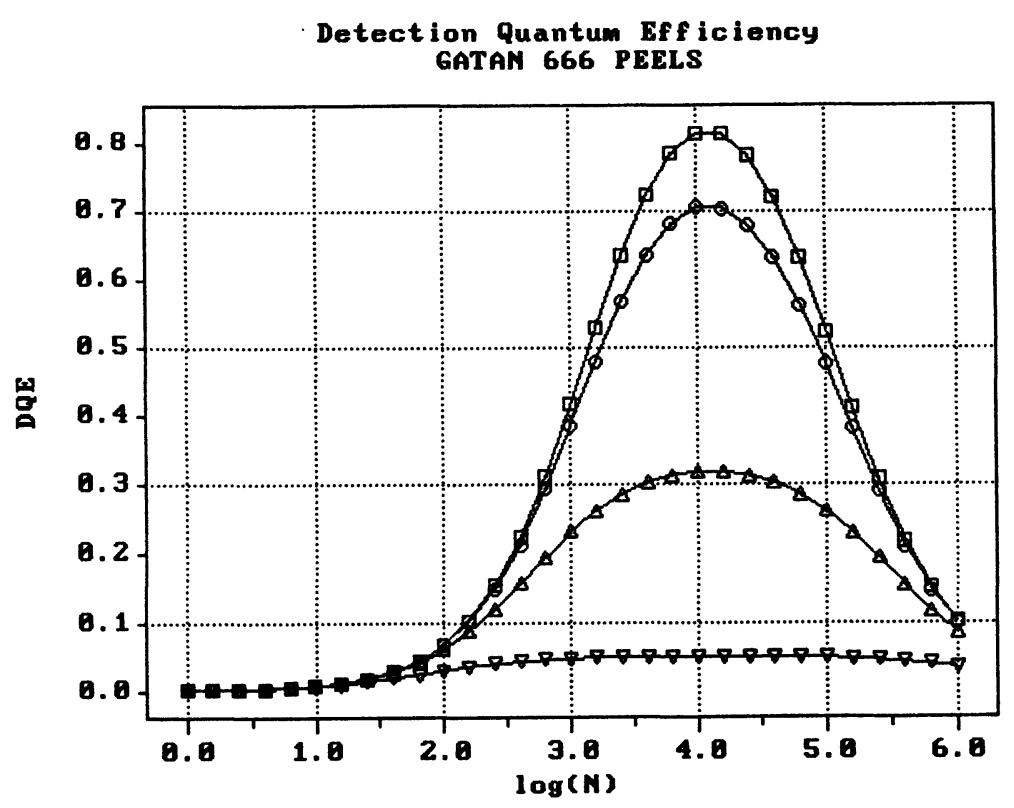

Fig. 3. $-\operatorname{DQE}(N)$ of the GATAN 666 PEELS for various count rates: (口) $S^{\prime}=10^{5} \mathrm{cts} / \mathrm{s},(\circ) S^{\prime}=10^{2} \mathrm{cts} / \mathrm{s}$, $(\Delta) S^{\prime}=10 \mathrm{cts} / \mathrm{s},(\nabla) S^{\prime}=1 \mathrm{cts} / \mathrm{s} . S^{\prime}$ is the count rate including the background signal.

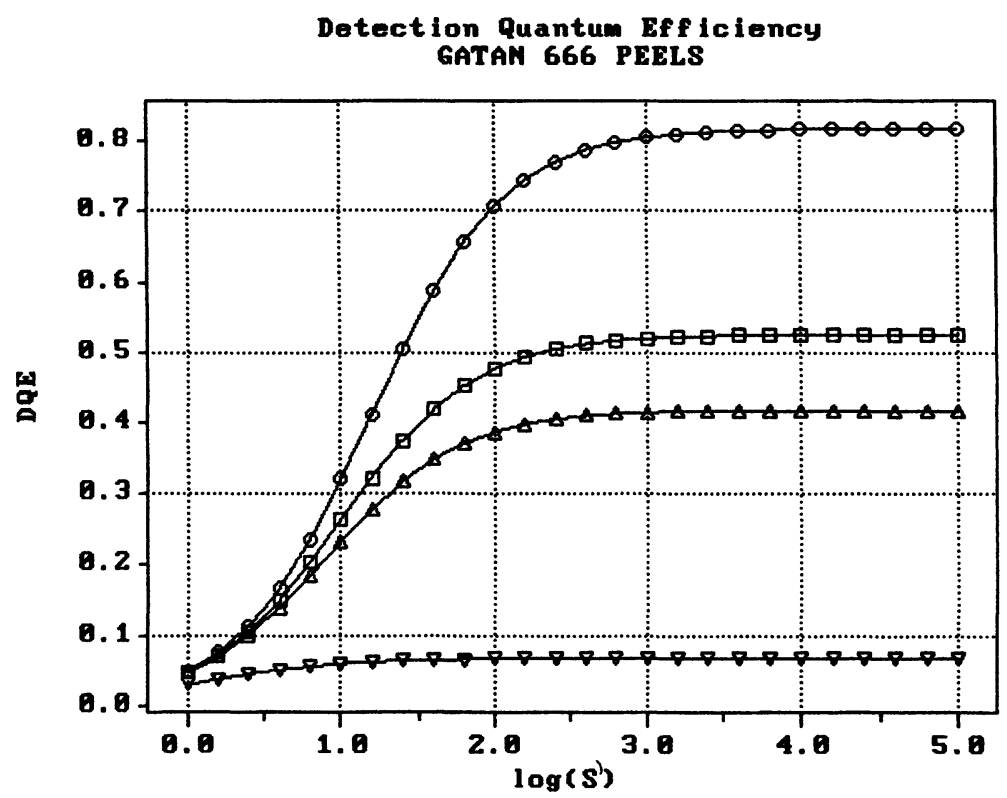

Fig. 4. $-\operatorname{DQE}\left(S^{\prime}\right)$ for various accumulated counts $N$. (ㅁ) $N=10^{5} \mathrm{cts} / \mathrm{s},($ ( ) $N=12300 \mathrm{cts} / \mathrm{s},(\Delta) N=$ $1000 \mathrm{cts} / \mathrm{s},(\nabla) N=100 \mathrm{ct} / \mathrm{s}$. 
SNR for background $=2 *$ signal GaTAN 666 PEELS

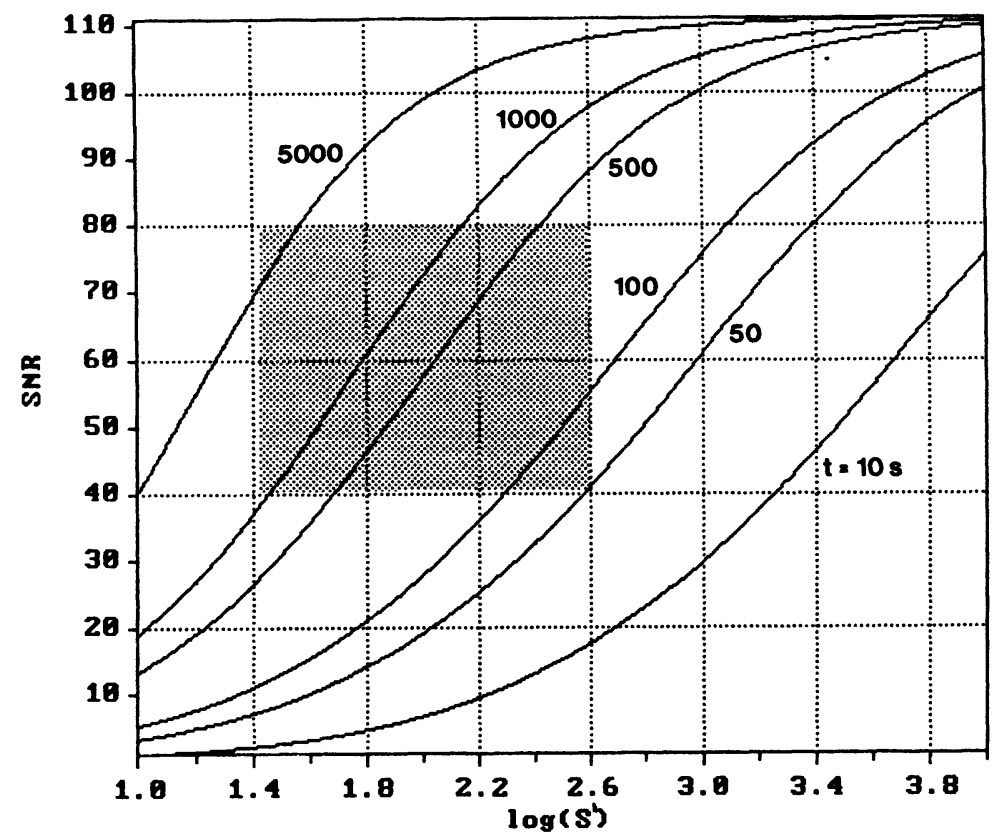

Fig. 5. $-\operatorname{SNR}\left(S^{\prime}\right)$ for $k=2$ at various dwell times $(10 \mathrm{~s}<t<5000 \mathrm{~s})$. The range of ECOSS experiments is marked.

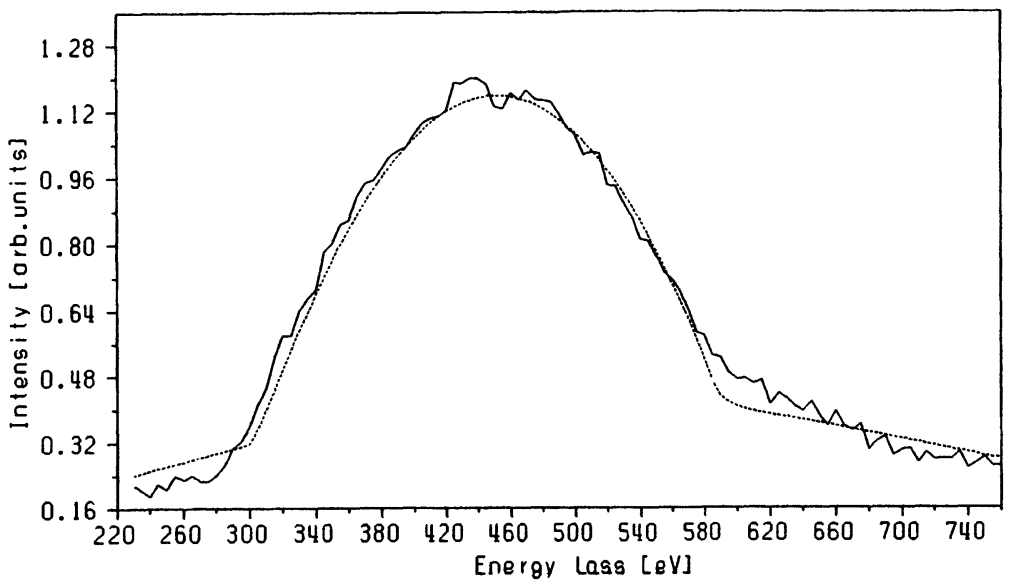

Fig. 6. - Compton spectrum of polycrystalline aluminium obtained from figure 1 after linear correction using equation (8) and background subtraction. Theoretical prediction superimposed (dashed line). 


\section{Conclusion.}

It was shown that ECOSS experiments can be done with the GATAN 666 PEELS. However, the expected rates coincide with the onset of a drop in the DQE induced by dark current. According to their better performance (see the Tab. in Sect. 3), the possibility to use CCDs instead of PDAs appears very attractive for future work with low count rates such as Compton scattering.

\section{Acknowledgements.}

This work was sponsored by the Austrian Fonds zur Förderung der Wissenschaftlichen Forschung, project P7432-Phy.

\section{References}

[1] Williams B.G., Egerton R.F., Chem. Phys. Lett. 88 (1982) 95-97.

[2] Schattschneider P., Pongratz P., Proc. 12. Werner Brandt Int. Conf. on the Penetration of Charged Particles in Matter, 35-60. 1989 (San Sebastian, Spain).

[3] Jonas P., SCHATTSChNEIDER P., PONGRATZ P., Proc. XII. ICEM Seattle (1990).

[4] SCHATTSCHNEIDER P., HOHENEGgeR H., Analytical Electron Microscopy - 1987, D.C. Joy Ed. (San Francisco Press, 1987) 270-74.

[5] Williams B.G., Uppal M.K., BRYdson R.D., Proc. R. Soc. Lond. A 409 (1987) 161-176.

[6 Williams B.G., Parkinson M.P., EcKhardT C.J., Thomas J.M., Chem. Phys. Lett. 78 (1981) 434-438.

[7] Williams B.G., SParrow T.G., EgerTON R.F., Proc. R. Soc. Lond. A 393 (1984) 409-422.

[8] Vasudevan S., Rayment T., Williams B.G., Holt R., Proc. R. Soc. Lond. A 391 (1984) 109-124.

[9] see for instance the proceedings of the NSF/CNRS Workshop on Electron Beam Induced Spectroscopies, Aussois 1988, and references therein.

[10] BATSON P., Rev. Sci. Instrum. 59 (1988) 1132-8.

[11] EgerTON R.F., J. El. Microsc. Tech. 1 (1984) 37-52.

[12] STRAUSS M.G., NADAY I., SHERMAN I.S., ZALUZEK N.J., Ultramicroscopy 22 (1987) 117-24.

[13] ZALUZEK N.J., STRAUSS M.G., in [9] pp. 190-95.

[14] EgerTon R.F., EELS in the Electron Microscope (New York: Plenum Press, 1986) pp. 357-361.

[15] KRIVANEK O.L., AHN C.C. and KEENEY R.B., Ultramicroscopy 22 (1987) 103-116.

[16] BERGER S.D., MCMULLAN D., in [9] pp. 182-84.

[17] Photometrics Ltd. CCD selection chart, May 1989.

[18] VG Microscopes technical specification ELS 6000P.

[19] see, e.g., SCHATTSCHNEIDER P., Fundamentals of Inelastic Electron Scattering, $22 \mathrm{ff}$ (Wien, New York: Springer-Verlag, 1986). 